NISTIR 5022

\title{
RangeCAD and the NIST RCS Uncertainty Analysis
}

Richard L. Lewis

Lorant A. Muth

Ronald C. Wittmann

QC

100

.056

\# 5022 



\section{RangeCAD and the NIST RCS Uncertainty Analysis}

\section{Richard L. Lewis}

Lorant A. Muth

Ronald C. Wittmann

Electromagnetic Fields Division

Electronics and Electrical Engineering Laboratory

National Institute of Standards and Technology

Boulder, Colorado 80303-3328

August 1994

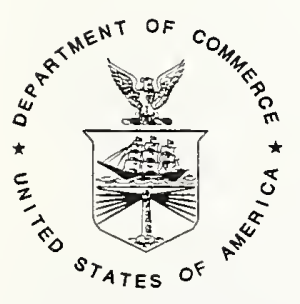

U.S. DEPARTMENT OF COMMERCE, Ronald H. Brown, Secretary TECHNOLOGY ADMINISTRATION, Mary L. Good, Under Secretary for Technology NATIONAL INSTITUTE OF STANDARDS AND TECHNOLOGY, Arati Prabhakar, Director 



\title{
RangeCAD and the NIST RCS Uncertainty Analysis
}

\author{
Richard L. Lewis, Lorant A. Muth and Ronald C. Wittmann \\ Electromagnetic Fields Division \\ National Institute of Standards and Technology \\ Boulder, Colorado 80303-3328
}

We discuss the salient features of a computer program, RangeCAD, and then translate the program's output into a catalogue of radar cross-section (RCS) uncertainties. This specific catalogue was developed by NIST to standardize RCS uncertainty computations at the various RCS measurement sites. We check uncertainty estimates generated by RangeCAD against alternative formulations that approximate equivalent uncertainty specifications. Based on this comparison, we conclude that the uncertainty estimates generated by RangeCAD provide realistic values for the NIST RCS uncertainty analysis.

Key words: error catalogue; measurement error; radar; radar cross section; RCS; radar signal analysis; uncertainty analysis

\section{Introduction}

The National Institute of Standards and Technology (NIST) has been assigned the task of evaluating radar cross section (RCS) measurement uncertainty at various Department of Defense sites. As a part of this effort, NIST has been studying a computer program, RangeCAD, that was developed for the United States Air Force to assist in making uncertainty predictions.

The computer program RangeCAD is essentially a sophisticated worksheet for converting estimated measurement-system parameter uncertainties into an overall estimate of the uncertainty in predicted RCS level, using a comprehensive model of the RCS measurement system for making these predictions. The user independently specifies values for the measurement system parameters along with their associated variances. Fortunately, as a part of RangeCAD's development, a fairly extensive error-component database was compiled, consisting of measured system parameters and the associated variance in these measured values. The existing database characterizes measurements taken on radars at the 46th Test Group's RATSCAT division, Holloman Air Force Base, and at the contractor's Electronic Test Site. A sample plan for taking measurements to update this database or to customize it for other RCS facilities is described in the program users manual.

RangeCAD is capable of fully accounting for all target-ground reflections occurring within the specified range-gate window, incorporating the effects of uncertainties attributable to the transmitted waveform, the antenna pattern, and the receiver in its predictions. It can also provide a premeasurement diagnosis of the measurement setup. The program's principal limitation is that a model of the target's radar cross section is 
needed in order to determine the measured RCS of the target. This is handled by utilizing the program to construct a dynamic-range model of the measurement system, from which the expected mean and variance of the measured RCS can be predicted for any measurement level. Construction of the dynamic-range model is accomplished by replacing the target with a single point scatterer whose scattering amplitude varies over the expected measurement range. The field taper across the spatial extent of the target remains unaccounted for in this implementation. However, the program permits field-taper mapping by displacing the point scatterer from the center of the target mount, thereby enabling a dynamic-range model to be produced for any desired location within the target zone's spatial extent.

The output from RangeCAD can be incorporated into the NIST RCS uncertainty analysis, which is a table of significant error sources designed to facilitate insight into RCS uncertainty computations and to standardize these calculations. The estimates of RCS uncertainty generated by RangeCAD can be compared to uncertainty estimates obtained from alternative formulations (see below). In spite of differences between underlying assumptions, the results generated by RangeCAD and the alternative formulations were consistent within an acceptable margin, an attribute which tended to lend credibility to RangeCAD.

\section{Salient Features of RangeCAD}

RangeCAD is designed to model a ground-bounce RCS antenna range for targets consisting of an ensemble of spatially-distributed scattering centers (point scatterers). Use of such models to simulate extended targets has proven very successful [1]. The RangeCAD program fully accounts for direct and ground-reflection ray paths from the antenna to each point scatterer on the target and back again. Also, in the case of targets specified using GTD (Geometrical Theory of Diffraction), coupling between the target and the ground is accounted for by adjusting the target's scattering-point locations to correspond to a ground-bounce ray-path excitation. The round-trip propagation-path length is obtained for each scattering point along all ray paths in order to determine the corresponding excitation amplitudes on the transmitted waveform's envelope. The program determines the received voltage from each scattering point as a function of the transmitted waveform amplitude, the antenna gain in the direction of the scatterer, the range propagation gain, the reflected amplitude and phase of the scatterer, and coherent-integration Doppler-mismatch loss. The instantaneous received voltages from each point scatterer within the receiver's range-gate window are summed along with the background clutter, and then a receiver linearity correction is applied to obtain the detected signal. A separate calculation of additive noise is made to include receiver noise, transmitter noise, antenna noise, and rf interference.

The instantaneous received power from a single scatterer [2] is given by

$$
P_{R}(t)=P_{T}(t-\tau) \frac{G^{2} \lambda^{2}}{(4 \pi)^{3} r^{4}} \sigma
$$

where $\tau=2 r / c$ is the scatterer's round trip delay time, $r$ is the range to the scatterer, $c$ is the propagation velocity, $\lambda$ is the wavelength, $G$ is the one-way antenna power gain in 
the direction of the scatterer, $P_{T}(t)$ is the instantaneous transmitted power, and $\sigma$ is the scatterer's RCS. A ground-reflection factor must be inserted when the propagation path intersects the ground. The instantaneous receiver response (video signal) to the point scatterer is given [2] (also see Appendix A) by

$$
Z_{R}(t)=\chi(t-\tau) D(\nu) \frac{G \lambda}{(4 \pi)^{\frac{3}{2}} r^{2}} \sigma^{\frac{1}{2}}
$$

which has a structure similar to eq (1). Here, $D(\nu)$ represents Doppler integrationmismatch loss for a Doppler-shift frequency $\nu$, while $\chi(t)$ (the ambiguity function) represents a convolution between the receiver impulse response and the pulse shape (envelope) of the transmitted signal. Such a convolution is included in RangeCAD's waveform model. For targets consisting of an ensemble of scatterers, the individual complex video signals are summed [2]. After a receiver linearity correction is made in RangeCAD, the resulting sum is squared and then combined with an additive noise term to obtain the composite video power sampled by the receiver [2,3].

In RangeCAD, the power received from a target is calculated as a function of that target's ideal RCS. The measured RCS of a target is obtained from the ratio of the target's received power to the calibration standard's received power times the calibration standard's ideal (theoretical) RCS $\sigma_{s}^{(c)}$. The measurement error is then obtained as the difference between the target's ideal and measured RCS. The measured RCS $\sigma_{m}$ is given by

$$
\sigma_{m}=\frac{P_{t}}{P_{s}} \sigma_{s}^{(c)}
$$

Here, $P_{t}$ and $P_{s}$ represent calculated received power from the target and the standard, respectively, based on a simulation of the measurement system (including system errors).

Program RangeCAD supports ideal RCS calculations for three built-in target models. For two of these models, a thin flat plate and a finite-length circular cylinder, the ideal RCS is calculated using GTD analysis. For the third model, a sphere, the ideal RCS is calculated using the Mie series. In addition to these theoretical models, the ideal RCS for an arbitrary target may be specified by an array of point scatterers, in which case the user must specify the scattering amplitude, phase, and position of each point scatterer.

A single point scatterer may be specified to simulate a probe target. For more complex targets, a subsidiary program is available to generate a planar array of point scatterers corresponding to a given far-field pattern, such as a computed scattered field. In principle, a planar array of point scatterers could be generated from measured bistatic RCS data to represent an arbitrary target. RangeCAD would then be able to give a first-order approximation to the measurement uncertainty. Generally, such a procedure is impractical due to the excessive amount of data required.

As a consequence, the normal use of RangeCAD is to obtain a family of simulated RCS measurements from a single point-scatterer target whose scattering amplitude varies over the measurement system's dynamic range. Thus, RangeCAD's primary usefulness lies in enabling the user to evaluate the uncertainty in an actual RCS measurement from a lookup table incorporating a dynamic-range model of the measurement system. In this 
role, RangeCAD can evaluate the effect of system parameter changes on RCS measurement systems and provide premeasurement diagnoses. A separate postmeasurement processing program should be used to compensate actual RCS measurements for recognized systematic errors, such as receiver nonlinearity, and the benefit of these corrections incorporated into RangeCAD's input parameter set.

The uncertainty in a RCS measurement can be expressed in decibels as

$$
10 \log \left(1 \pm \sqrt{\mu}_{\sigma} / \sigma\right),
$$

where $\mu_{\sigma}$ is the total RCS variance and $\sigma$ is the associated RCS level (nominally, $\sigma$ either denotes the measured RCS of the calibration standard or the target). If the RCS is a function of a set of $N$ parameters $x_{i}$, and $\mu_{x_{i}}$ is the variance of $x_{i}, i=1, N$, then the total RCS variance [4] is given by

$$
\mu_{\sigma}=\sum_{i=1}^{N}\left|\frac{\partial \sigma}{\partial x_{i}}\right|^{2} \mu_{x_{i}}
$$

RangeCAD only includes those parameters in the above sum which have been assigned nontrivial variance values by the user. In eq (5), the coefficient of $\mu_{x_{i}}$ is called the sensitivity, and the product of that sensitivity with $\mu_{x_{i}}$ represents a partial RCS variance due to the uncertainty in $x_{i}$. The partial derivatives are calculated using a simple two-term, divided-difference formula. That is, the derivative is obtained by calculating the RCS at a small increment above and below the mean value for the parameter, and dividing the difference between these two results by two times that increment. In terms of Lagrange interpolation, this approximation to the first derivative is correct through to the third derivative [5]. As a result, whatever RCS functional dependence a user might specify for a parameter, that dependence is fully taken into account by the sensitivity calculation.

RangeCAD was designed so that separate data-input files are specified for the measurement of calibration standards and the measurement of targets. This feature is normally used to change the rf attenuation between the calibration measurement and the target measurement. Radar parameter measurements have been carried out at VHF, UHF, L, S, $\mathrm{C}$, and $\mathrm{X}$ band frequencies, and corresponding RangeCAD data files have been developed [6] for use at the contractor's Electronic Test Site and at RATSCAT. Generally, mean parameter values are given, while the variances represent the measurement uncertainty. Only the variances of those parameters which resulted in significant RCS uncertainties are retained in the data files.

Although RangeCAD was originally designed for use at RATSCAT, the program's modular nature allows it to be readily adapted for use at other sites. For instance, the U.S. Navy's Santa Cruz Radar Imaging Facility (SCRIF) has the same model radar as at RATSCAT, so RangeCAD could be put to use at SCRIF using very similar input data as that generated at RATSCAT. Step-by-step procedures for carrying out and verifying parameter measurements are given in the RangeCAD user's manual [6], so the database can readily be customized. For instance, the basic parameter set has been modified at RATSCAT to characterize three different measurement systems. A significant feature enabling RangeCAD to be used at other sites is the separate data-input file structure mentioned above. For instance, at SCRIF the calibration standard is a dihedral located 
across a valley, whereas the targets are ships, so for this facility the calibration measurement's ground-plane parameters would be set to free-space values, while for the target's measurement they would simulate the sea. ${ }^{1}$

\section{RangeCAD and the NIST RCS Uncertainty Analysis}

The NIST uncertainty analysis for RCS measurements was created to identify uncertainty categories that would apply throughout the RCS measurement community [7]. Subsidiary uncertainty tables specific to individual sites can easily be incorporated within this framework. Table 1 presents an example of the NIST RCS uncertainty analysis. Here, separate tables are provided for the target and calibration measurements. Partial RCS uncertainties are combined on the last line of each table by converting the partial uncertainties (expressed in decibels) to variances and then summing these variances to obtain the total RCS variance. The overall RCS uncertainty in decibels is then obtained from the total RCS variance as shown in eq (4).

Predicted RCS uncertainties, obtained from RangeCAD using an S-band data set, are incorporated into the NIST RCS uncertainty analysis framework as shown in Table 1. The numerical values presented in the table should be considered illustrative rather than applicable to any specific measurement. Moreover, the computed uncertainty values depend upon the RCS level. In the case of Table 1, the values presented assume distinct calibration and target RCS levels of $10 \mathrm{dBsm}$ and $-20 \mathrm{dBsm}$, respectively. Obviously, a number of tables would be needed to fully characterize a RCS measurement. The uncertainty associated with specific RCS measurement levels could then be determined from the appropriate table. Alternately, a subsidiary program is available with RangeCAD for automating this process.

In the following, each entry in the NIST RCS uncertainty-analysis table is examined, and RangeCAD's capability for estimating the partial RCS uncertainty is indicated where applicable. Alternative formulations for validating specific results are also discussed.

$1.1 \& 2.1$ Average Illumination: Tracking and pointing uncertainty constitutes this error category [7]. Specifying allowable variances in the target's position variables enables RangeCAD to calculate the uncertainty directly. Alternately, if an angular tracking uncertainty $\Delta$ is specified, then (see Appendix B) the RCS uncertainty in decibels is $-20 \log \left[1-\sin ^{2}\left(\frac{\pi}{2} \Delta / \theta_{0}\right) \operatorname{sinc}(2 X)\right]$. Here, $\operatorname{sinc} X=\sin X / X$, where $X=\frac{\pi}{4} \theta_{1} / \theta_{0}, \theta_{1}$ is the angular span of the target, and $\theta_{0}$ is the half-power beamwidth. Alternately, we can obtain $X$ from a measurement of the FieldTaper (cf. Near Field below). Our calculations in the table use the values FieldTaper $=0.5 \mathrm{~dB}, \theta_{0}=2.5^{\circ}$, and $\Delta=0.1^{\circ}$.

$1.2 \& 2.2$ Background/Target Interactions: Target/pylon interaction and clutter data are supplied to RangeCAD by files TGT_MOUNT.DAT and CAL_MOUNT.DAT, which contain tabular frequency and/or azimuth dependent values which can be used for interpolation. Target-to-pylon RCS ratios as small as $10 \mathrm{~dB}$ can result in a measurement

1 The prospective user should verifythat a program modification suggested by NIST has been implemented so that the free-space ground-plane response will be correct. 
uncertainty of $3 \mathrm{~dB}$. As the target RCS approaches the pylon RCS destructive interference effects can be dramatic [8]. Numerical data for specifying file values are not available to NIST.

1.3 \& 2.3 Cross-Polarization Effects: Measurements carried out [9] at RATSCAT in 1988 indicated a cross-polarization isolation of less than $-30 \mathrm{~dB}$. The values in the table assume a cross-polarization level around $-40 \mathrm{~dB}$ with a non-depolarizing standard and a depolarizing target. RangeCAD may be used directly to obtain the uncertainty by specifying a polarization-angle variance in RangeCAD's antenna-parameter file. Alternately [7], as in the table, assuming a cross-polarization level of $-\epsilon \mathrm{dB}$ and a depolarizing target produces an uncertainty of $20 \log \left(1+2 \times 10^{-\epsilon / 20}\right)$, while a non-depolarizing target produces an uncertainty of $20 \log \left(1+10^{-\epsilon / 10}\right)$.

$1.4 \& 2.4$ Drift: Tests at RATSCAT carried out in 1991 [6] and in 1988 [9] indicated propagation path drifts of about $0.5 \mathrm{~dB}$ over an hour's time. Periodic recalibration using secondary standards limits expected drift. The table assumes negligible drift during calibration.

1.5 \& 2.5 Frequency: As discussed above, RangeCAD obtains the RCS sensitivity to a parameter by recalculating the RCS at small increments above and below the parameter's mean value. For RCS frequency response, RangeCAD supports specifying target-pylon clutter as a function of frequency, thereby explicitly enabling target/targetmount frequency effects to be modeled. Inasmuch as frequency variance is limited to the bandwidth squared, except for unusual cases the expected contribution to RCS variance from gradients in the received-signal amplitude as a function of frequency should be small. However, a small change in frequency can result in a significant phase change between scatterers, which could lead to a large RCS variance. For example, two scatterers separated in range by $L$ will undergo a $2 \pi$ relative phase change in RCS due to a frequency change $\Delta f$, where $\Delta f / f=\lambda /(2 L)[2]$. For $L / \lambda=125$, a $0.2 \%$ relative frequency shift results in a $180^{\circ}$ phase shift, which could cause the combined response to vary from a maximum to a null.

$1.6 \& 2.6$ Integration: Both A/D quantization loss and Doppler integration mismatch loss fit this category. Integration uncertainty is obtained by summing the individual partial RCS variances and using eq (4) to convert the result to a partial RCS uncertainty expressed in decibels. Assuming an 8-bit A/D converter, the quantization loss is given by $-20 \log (255 / 256) \approx 0.03 \mathrm{~dB}$. Doppler integration-mismatch loss is given [6] (cf. (A-11)) by

$$
-20 \log \left(\frac{\sin \frac{N \phi}{2}}{N \sin \frac{\phi}{2}}\right)
$$

The pulse phase shift $\phi=2 \pi \times$ width $\times$ AzimuthRotationRate $/(\lambda \times P R F)$, where width denotes the target's (electrical) span, $\lambda$ the wavelength, $P R F$ the pulse repetition frequency, and $N$ the number of integrated pulses. Normally, Doppler integration-mismatch loss can be expected to be small.

$1.7 \& 2.7$ I/Q Imbalance: The table directly uses RangeCAD's predictions. Target 
and calibration-standard uncertainty calculations are independently carried out at distinct RCS levels. Gain imbalance and phase imbalance contribute to this category. The individual partial RCS variances are summed and then eq (4) is used to convert the result to a partial RCS uncertainty expressed in decibels.

$1.8 \& 2.8$ Near Field: Field taper across the target constitutes this error category. Uncertainty may be obtained directly from RangeCAD using the program's probe option to predict RCS gain reduction in the target volume. Alternately, uncertainty can be determined from geometry and the antenna's half-power beamwidth $\theta_{0}$ or from field-taper measurements. For instance, probe measurements [9] in 1988 showed horizontal and vertical field tapers of $0.5 \mathrm{~dB}$. Transmitting antenna gain and receiving antenna gain doubles the potential error. Assuming a cosine-squared antenna pattern and a target that is a linear array of equal-amplitude uniformly-distributed point scatterers, the RCS uncertainty [10] is $-20 \log [(1+\operatorname{sinc}(2 X)) / 2]$, where $\operatorname{sinc} X=\sin X / X$. Here, $X=\frac{\pi}{4} \theta_{1} / \theta_{0}$, where $\theta_{1}$ is the angle subtended by the target at the antenna. Alternately, one may obtain $X$ by solving the equation $-20 \log (\cos X)=$ FieldTaper. The latter option is used in the table, where FieldTaper $=0.5 \mathrm{~dB}$, in accord with measured values reported above.

1.9 \& 2.9 Noise/Background: The table directly uses RangeCAD's predictions. Target and calibration-standard uncertainty calculations are independently carried out at distinct RCS levels. Noise, background magnitude, clutter amplitude, background phase, clutter phase, and ground-plane roughness all contribute to this category. The partial RCS variances are summed and then eq (4) is used to obtain this category's combined partial RCS uncertainty.

$1.10 \& 2.10$ Nonlinearity: RangeCAD predictions are used directly in the table. Target and calibration-standard uncertainty calculations are independently carried out at distinct RCS levels. Receiver nonlinearity variance and a number of waveform parameter variances all contribute to this category. The individual partial RCS variances are summed and then eq (4) is used to obtain the combined partial RCS uncertainty for this category. Inasmuch as a point-scatterer target is used, the waveform-parameter variances should be set equal to the square of the maximum variation that a point scatterer would experience on a range walk over the extent of the actual target.

1.11 \& 2.11 Range: Assuming that the target range uncertainty is $\Delta R$, the RCS uncertainty is $-40 \log (1-\Delta R / R)$, which is usually negligible. RangeCAD may be used directly for this calculation.

$1.12 \& 2.12$ Target Orientation: Uncertainty may be obtained from RangeCAD by specifying allowable variances in the target's angular positioning variables. Normally, this uncertainty contribution can be expected to be small.

2.13 Computational Error: Numerical computation error for standard target's RCS, including manufacturing defects. The table's estimate of $0.5 \mathrm{~dB}$ is consistent with RCS uncertainty estimates [9] for cylinders and with uncertainties in numerical computations obtained at NIST.

1.13 Calibration Uncertainty: Overall calibration uncertainty from 2.14. 
$1.14 \& 2.14$ Overall Uncertainty: The partial RCS variances are obtained for each category and then summed to obtain the total RCS variance. The overall uncertainty expressed in decibels is then obtained using eq (4).

\section{Conclusion}

Program RangeCAD's formulation for the radar signal received from an illuminated target agrees in form with established expressions for representing the received video signal of a monostatic radar due to if illumination of an ensemble of point scatterers. We have pointed out significant features of RangeCAD and discussed the possibility of using program output to ease the experimentalist's task of constructing an RCS uncertainty analysis. In this regard, when using dynamic-range modeling to evaluate RCS uncertainty it is apparent that some partial variances should be exaggerated (for example, waveform parameter variances should be augmented to include the range of waveform values experienced over the downrange extent of the target). Also, some variances (for example, near field or field taper) are readily evaluated by alternative expressions whose use may be more convenient. The RangeCAD program is applicable to or can be adapted to a wide variety of RCS ranges. Although RangeCAD is not a panacea for determining RCS uncertainty, it can be viewed as a useful tool to steer the experimentalist into considerations that might otherwise be overlooked, and it could become a model for producing comparable RCS uncertainty estimates at applicable ranges. Moreover, the task of constructing the RangeCAD parameter tables enables the user to track equipment stability over time, and could lead to RCS measurement error detection (as opposed to RCS uncertainties) which would require compensation by a post-measurement processing program. Finally, using RangeCAD to carry out premeasurement diagnosis might avoid wasting valuable measurement time by detecting otherwise overlooked problems.

The authors would like to express their appreciation to Barry Stroman, Martin Marietta Orlando Aerospace, for helpful discussions regarding RangeCAD. 


\section{References}

[1] Mitchell, R. L. Models of extended targets and their coherent radar images. Proc. IEEE. 62 (6): 754-758; 1974 June.

[2] Mitchell, R. L. Radar signal simulation. Artech House, Inc.; 1976.

[3] Minkoff,J. Signals, noise, and active sensors. Wiley \& Sons; 1992.

[4] Taylor, B. N.; Kuyatt, C. E. Guidelines for evaluating and expressing the uncertainty of NIST measurement results. Natl. Inst. Stand. Technol. (U.S.) Tech. Note 1297; 1993 January.

[5] Hildebrand, F. B. Introduction to numerical analysis. McGraw-Hill; 1956.

[6] Range_CAD user's manual, Range_CAD programmer's manual, and RCS measurement error source analysis program phase III \& phase IV final technical report; 1991 April. and Range_CAD user's manual, revision 2; 1992 September: distribution by Holloman Air Force Base.

[7] Wittmann, R. C.; Francis, M. H.; Muth, L. A.; Lewis, R. L. Proposed uncertainty analysis for RCS measurement. Natl. Inst. Stand. Technol. (U.S.) NISTIR 5019; 1994 January.

[8] Wollny, W. T. RCS errors due to target support structure. Quick Reaction Corp. P.O. Box 96, Gilroy CA, 95020, Proc. AMTA; 1988.

[9] Bistatic radar cross section measurement considerations. DynCorp; 1988 September: limited distribution by Holloman Air Force Base.

[10] Welsh, B. M.; Link, J. N. Accuracy criteria for radar cross section measurements of targets consisting of multiple independent scatterers. IEEE Trans. Antennas Propagat. AP-36(11): 1587-1593; 1988 November. 
Table 1. Typical RCS uncertainties based in part on RangeCAD data

$\begin{array}{rlrr} & \text { MEASUREMENT UNCERTAINTIES } & +\mathrm{dB} & -\mathrm{dB} \\ 1.1 & \text { Average illumination } & 0.03 & -0.03 \\ 1.2 & \text { Background/target interaction } & ? & ? \\ 1.3 & \text { Cross polarization } & 0.16 & -0.17 \\ 1.4 & \text { Drift } & 0.50 & -0.57 \\ 1.5 & \text { Frequency } & 0.00 & 0.00 \\ 1.6 & \text { Integration } & 0.03 & -0.03 \\ 1.7 & \text { I/Q imbalance } & 0.67 & -0.79 \\ 1.8 & \text { Near field } & 0.33 & -0.35 \\ 1.9 & \text { Noise/background } & 0.65 & -0.76 \\ 1.10 & \text { Nonlinearity } & 0.07 & -0.07 \\ 1.11 & \text { Range } & 0.00 & 0.00 \\ 1.12 & \text { Target orientation } & 0.00 & 0.00 \\ 1.13 & \text { Calibration uncertainty (2.14) } & 0.83 & -1.02 \\ & & & \\ 1.14 & \text { Overall uncertainty } & 1.29 & -1.85\end{array}$

CALIBRATION UNCERTAINTIES $+\mathrm{dB}-\mathrm{dB}$

1.1 Average Illumination

1.2 Background/target interaction

1.3 Cross polarization

$0.03-0.03$

1.4 Drift

1.5 Frequency

? ?

$0.00 \quad 0.00$

$0.00 \quad 0.00$

1.6 Integration

$0.00 \quad 0.00$

1.7 I/Q imbalance

$\begin{array}{ll}0.03 & -0.03\end{array}$

1.8 Near field

$\begin{array}{ll}0.66 & -0.77\end{array}$

1.9 Noise/background

$\begin{array}{ll}0.16 & -0.17\end{array}$

1.10 Nonlinearity

$0.12-0.13$

1.11 Range

$0.05 \quad-0.05$

1.12 Target orientation

$0.00 \quad 0.00$

1.13 Computational error

$0.00 \quad 0.00$

$\begin{array}{ll}0.50 & -0.57\end{array}$

1.14 Overall calibration uncertainty

$\begin{array}{ll}0.83 & -1.02\end{array}$ 


\section{Appendix A. Radar Signal Representations}

Here we present a brief review of the mathematical representation of the signal received by a radar and the associated receiver response. Additional details may be found in [2]. The theory's application to waveform analysis and range-Doppler resolution is further discussed in [3].

Let $\psi_{T}(t)$ denote a transmitted rf signal at time $t$ having power $P_{T}(t)=|\psi(t)|^{2}$, and let $\gamma$ denote the complex reflection coefficient of a point target (i.e., a target that is small compared to a resolution cell) such that the point target's radar cross section $\sigma=|\gamma|^{2}$. Then the signal received by the radar from the scatterer $\gamma$ is

$$
\psi_{R}(t)=\psi_{T}(t-\tau(t)) \frac{G \lambda}{(4 \pi)^{\frac{3}{2}} r^{2}} \gamma,
$$

where $\lambda$ is the wavelength, $r$ is the range, $G$ is the one-way antenna gain in the direction of the scatterer, and $\tau(t)$ is the round-trip delay. The received power corresponding to the received signal $\psi_{R}(t)$ results in the familiar radar range equation

$$
P_{R}(t) \equiv\left|\psi_{R}(t)\right|^{2}=P_{T}(t-\tau(t)) \frac{G^{2} \lambda^{2}}{(4 \pi)^{3} r^{4}} \sigma .
$$

We now consider that the complex transmitted signal $\psi_{T}(t)$ can be decomposed into a sinusoidal function of the carrier frequency $f=c / \lambda$, where $c$ is the speed of light, and a complex modulation function containing only low frequencies, as $\psi_{T}(t)=\mu_{T}(t) e^{j 2 f t}$. We further consider that the target of interest consists of an ensemble of point scatterers. Modeling extended targets as a collection of point scattering centers is discussed in [1]. The received signal from the target is then given as a superposition of the component signals from each scattering center. We let $\gamma_{k}, G_{k}$, and $r_{k}$ denote the $k^{\text {th }}$ scatterer's complex reflection coefficient, gain, and range, respectively. The instantaneous round-trip delay can be expanded into a Taylor series as $\tau(t)=\tau_{k}-\nu_{k} t / f$, where $\tau_{k}=2 r_{k} / c$ is the delay for the $k^{t h}$ scatterer and $\nu_{k}=-2 \dot{r} / \lambda$ is the associated Doppler coefficient (this assumes the scatterer to be slowly traveling at a constant range rate $\dot{r}$ over some time interval of interest). Combining these specifications with our previous expression (A-1) for the signal received from a single point scatterer, we obtain [2]

$$
\psi_{R}(t)=e^{j 2 \pi f t} \sum_{k} V_{k} \mu_{T}\left(t-\tau_{k}\right) e^{j 2 \pi \nu_{k} t},
$$

where

$$
V_{k} \equiv\left[\frac{\lambda}{(4 \pi)^{\frac{3}{2}}}\right] \frac{1}{r_{k}^{2}} G_{k} \gamma_{k} e^{-j 2 \pi f \tau_{k}} .
$$

We now consider that the transmitted signal's complex modulation function consists of a train of $M$ short pulses, so that

$$
\mu_{T}(t)=\sum_{n=0}^{M-1} \mu_{P T}\left(t-n T_{r}\right),
$$


where $T_{r}$ is the pulse repetition period. Now if the individual pulse length $T_{P}$ is less than half $T_{r},\left|\tau_{k}\right| \ll M T_{r}$, and $\left|\nu_{k}\right| \ll 1 / T_{p}$, then we can introduce a much simplified analysis (thereby allowing us to evaluate each pulse's response as equivalent to that from a central pulse). Let $\tau_{k}^{\prime}=\tau_{k}\left(\bmod T_{r}\right)$ and $\nu_{k}^{\prime}=\nu_{k}\left(\bmod 1 / T_{r}\right)$. Consequently, $0 \leq \tau_{k}^{\prime}<T_{r}$ and $0 \leq\left|\nu_{k}^{\prime}\right|<1 / T_{r}$. Now for the moment, let us define $t$ as the time measured from the transmission of the $n^{t h}$ pulse and confine $t$ as $0 \leq t<T_{r}$. Then we can replace $t$ in our expression (A-3) for the received signal by $t+n T_{r}$ and write the received signal due to the $n^{\text {th }}$ pulse as

$$
\psi_{R}\left(t+n T_{r}\right)=e^{j 2 \pi f\left(t+n T_{r}\right)} \sum_{k} V_{k} \mu_{P T}\left(t-\tau_{k}^{\prime}\right) e^{j 2 \pi \nu_{k}^{\prime} n T_{r}},
$$

where we have dropped the essentially constant phase term $2 \pi \nu_{k}^{\prime} t$ (having bounded variation $\left.2 \pi\left|\nu_{k}^{\prime}\right| T_{p} \ll 2 \pi\right)$. The received signal is usually mixed down to video as the initial processing step in the receiver, so the received video signal can be written as

$$
\mu_{R}\left(t+n t_{r}\right)=\sum_{k} V_{k} \mu_{P T}\left(t-\tau_{k}^{\prime}\right) e^{j 2 \pi \nu_{k}^{\prime} n T_{r}} .
$$

If pulse compression is implemented next, then the output [2] corresponding to the $n^{\text {th }}$ pulse is given by

$$
Z_{n}(\tau)=\int_{0}^{T_{r}} \mu_{R}\left(t+n T_{r}\right) \mu_{P F}^{*}(t-\tau) d t=\sum_{k} V_{k} \chi_{P}\left(\tau-\tau_{k}^{\prime}\right) e^{j 2 \pi \nu_{k}^{\prime} n T_{r}} .
$$

where $\mu_{P F}^{*}(-t)$ is the impulse response of the receiver's filter (the receiver is assumed to be matched to a pulse-signal $\mu_{P F}(t)$ that resembles the pulse $\mu_{P T}(t)$ that is transmitted by the radar, and the asterisk denotes complex conjugation). Under our short-pulse assumption the filter response or ambiguity function $\chi_{P}(\tau)$ becomes independent of Doppler shift and reduces to the cross correlation

$$
\chi_{P}(\tau)=\int_{-\infty}^{+\infty} \mu_{P T}(t) \mu_{P F}^{*}(t-\tau) d t .
$$

Next, if coherent integration of $N$ pulses is implemented, $N \leq M$, then we would sum the individual pulse responses given in eq (A-8) over $n$. We note that at this point a tuning frequency could be introduced to compensate the received signal for Doppler shift; however, here we assume that the receiver is kept tuned to the transmitted frequency, which is applicable to airborne target simulation. If we also assume square-law detection, our final expression for the detected signal becomes [2,3]

$$
|Z(\tau)|^{2}=\left|\sum_{k} V_{k} \chi_{P}\left(\tau-\tau_{k}^{\prime}\right) \frac{1}{N} \sum_{n=0}^{N-1} e^{j 2 \pi \nu_{k}^{\prime} n T_{r}}\right|^{2}=\left|\sum_{k} V_{k} \chi_{P}\left(\tau-\tau_{k}^{\prime}\right) D\left(\nu_{k}\right)\right|^{2} \cdot \quad(A-10)
$$

It may be further noted that the absolute value of the Doppler fine structure coefficient $D(\nu)$ can be written as

$$
|D(\nu)|=\frac{\sin \left(N \pi \nu T_{r}\right)}{N \sin \left(\pi \nu T_{r}\right)} .
$$




\section{Appendix B. Estimation of Amplitude Taper Uncertainty}

Here we present a brief review of the estimated RCS uncertainty due to amplitude taper across a long target. In order to make the analysis tractable, a number of simplifying assumptions are made to characterize a generic RCS target. In particular, the target is modeled as a uniform distribution of equal amplitude point scatterers. A nonuniform distribution function can be used when apriori information is available about the target. Additional details concerning this analysis may be found in ref. [10].

The positions of the scatterers along the transverse dimensions of the target are described by a probability density function $p(\theta, \phi)$ with the radar located at the origin of a standard spherical coordinate system. The ratio of the measured RCS to the standard's RCS is given by

$$
\frac{\sigma_{m}}{\sigma_{s}^{(c)}}=\left|\frac{\psi_{R}^{m}(t)}{\psi_{R}^{s}(t)}\right|^{2} .
$$

Let the reference target consist of a single point scatterer $\gamma_{0}$. Then the ratio of received signals can be written as

$$
\frac{\psi_{R}^{m}(t)}{\psi_{R}^{s}(t)}=\frac{\sum_{k} \mu_{T}\left(t-\tau_{k}\right) \frac{r_{0}^{2}}{r_{k}^{2}} G_{k} \gamma_{k} e^{j 2 \pi\left[\left(\nu_{k}-\nu_{0}\right) t-f\left(\tau_{k}-\tau_{0}\right)\right]}}{\mu_{T}\left(t-\tau_{0}\right) G_{0} \gamma_{0}} .
$$

In most cases, the amplitude variations caused by differences in range between the scatterers is negligible. We further suppose that we can combine the amplitude modulation, the phase discrepancy between each scatterer, and the scatterer's reflection coefficient to produce an expected real reflection coefficient $\hat{\gamma}_{k}$. We further assume that the gain in the direction of the calibration target is unity. With these simplifications we can write the expected absolute value ratio of the received signals as

$$
\mathcal{E}\left\{\left|\frac{\psi_{R}^{m}(t)}{\psi_{R}^{s}(t)}\right|\right\} \simeq \frac{1}{\hat{\gamma}_{0}} \sum_{k} \hat{\gamma}_{k} G_{k} .
$$

If we further assume all scatterer's have the same cross-section and all of their positions are described by $p(\theta, \phi)$, then the expected absolute value ratio becomes

$$
\mathcal{E}\left\{\left|\frac{\psi_{R}^{m}(t)}{\psi_{R}^{s}(t)}\right|\right\} \simeq \frac{\hat{\gamma}_{1}}{\hat{\gamma}_{0}} \iint p(\theta, \phi) G(\theta, \phi) d \theta d \phi .
$$

In order to estimate the effects of amplitude taper, we will form the ratio of the measured signal to the signal obtained when the target is in the far field. We also assume a long thin target with the long dimension transverse to antenna boresight. Consequently, the double integration can be transformed to a single integral. Using the variable $y=r \theta$, where $r$ is the range to the target, we obtain

$$
\mathcal{E}\left\{\left|\frac{\psi_{R}^{m}(t)}{\psi_{R}^{f f}(t)}\right|\right\} \simeq \int_{L} p(y) G(y) d y
$$


where now $p(y)$ describes the point-scatterer probability density along the target length $L$. The ratio $\hat{\gamma}_{k} / \hat{\gamma}_{0}$ was eliminated by forming the ratio of the measured signal to the predicted far-field response [10].

Now if the center of the target is offset by $\delta y$ from antenna boresight and we assume a uniform point-scatterer probability density function, then

$$
\mathcal{E}\left\{\left|\frac{\psi_{R}^{m}(t)}{\psi_{R}^{f f}(t)}\right|\right\} \simeq \frac{1}{L} \int_{-\frac{L}{2}+\delta y}^{\frac{L}{2}+\delta y} G(y) d y .
$$

A simple antenna gain model that is easy to integrate is the cosine squared pattern. Accordingly, we assume $G(r \theta)=\cos ^{2}\left(\frac{\pi}{2} \frac{\theta}{\theta_{0}}\right)$, where $\theta_{0}$ is the antenna's half-power beamwidth. If we also assume $\delta y=r \Delta$, where $\Delta$ is the angular tracking error, and we define $L=r \theta_{1}$, where $\theta_{1}$ is the angle sustended by the target, then we obtain

$$
\mathcal{E}\left\{\left|\frac{\psi_{R}^{m}(t)}{\psi_{R}^{f f}(t)}\right|\right\} \simeq \frac{1}{\theta_{1}} \int_{-\frac{1}{2} \theta_{1}+\Delta}^{\frac{1}{2} \theta_{1}+\Delta} \cos ^{2}\left(\frac{\pi}{2} \frac{\theta}{\theta_{0}}\right) d \theta
$$

Carrying out the integration we readily obtain

$$
\mathcal{E}\left\{\left|\frac{\psi_{R}^{m}(t)}{\psi_{R}^{f f}(t)}\right|\right\} \simeq \frac{1}{2}\left[1+\operatorname{sinc}\left(\frac{\pi}{2} \frac{\theta_{1}}{\theta_{0}}\right) \cos \left(\pi \frac{\Delta}{\theta_{0}}\right)\right]
$$

where we have made use of the well known definition, $\operatorname{sinc} X=\sin X / X$.

The preceding expression (B-8) gives the effects of both amplitude taper across the target and pointing error. In carrying out an uncertainty analysis, we separate these two effects. We set $\Delta=0$ in eq (B-8), and express in decibels the total RCS near-field uncertainty as

$$
20 \log \frac{2}{1+\operatorname{sinc}\left(\frac{\pi}{2} \frac{\theta_{1}}{\theta_{0}}\right)} .
$$

We can also express in decibels the pointing uncertainty as

$$
20 \log \left(1+\operatorname{sinc}\left(\frac{\pi}{2} \frac{\theta_{1}}{\theta_{0}}\right) \sin ^{2}\left(\frac{\pi}{2} \frac{\theta_{1}}{\theta_{0}}\right)\right) .
$$

This expression was obtained by subtracting the expression for the effects of both amplitude taper and pointing error from the expression for amplitude taper alone and adding unity. 

. 